\title{
The Gold (AU)DRIPSS Framework: Factors for a Maturing Digital Preservation Strategy
}

\#r3a

National Digital Stewardship Alliance

October 18, 2018 


\section{Brett D. Currier}

Library Manager

Center for the Advancement of Data and Research in Economics

Federal Reserve Bank of Kansas City

Brett.currier@kc.frb.org
Courtney R. Butler

Data Curator

Center for the Advancement of Data and Research in Economics

Federal Reserve Bank of Kansas City

Courtney.Butler@kc.frb.org

The views expressed in this paper are those of the authors and do not necessarily reflect the official views of the Federal Reserve Bank of Kansas City or the Federal Reserve System. 
Why?

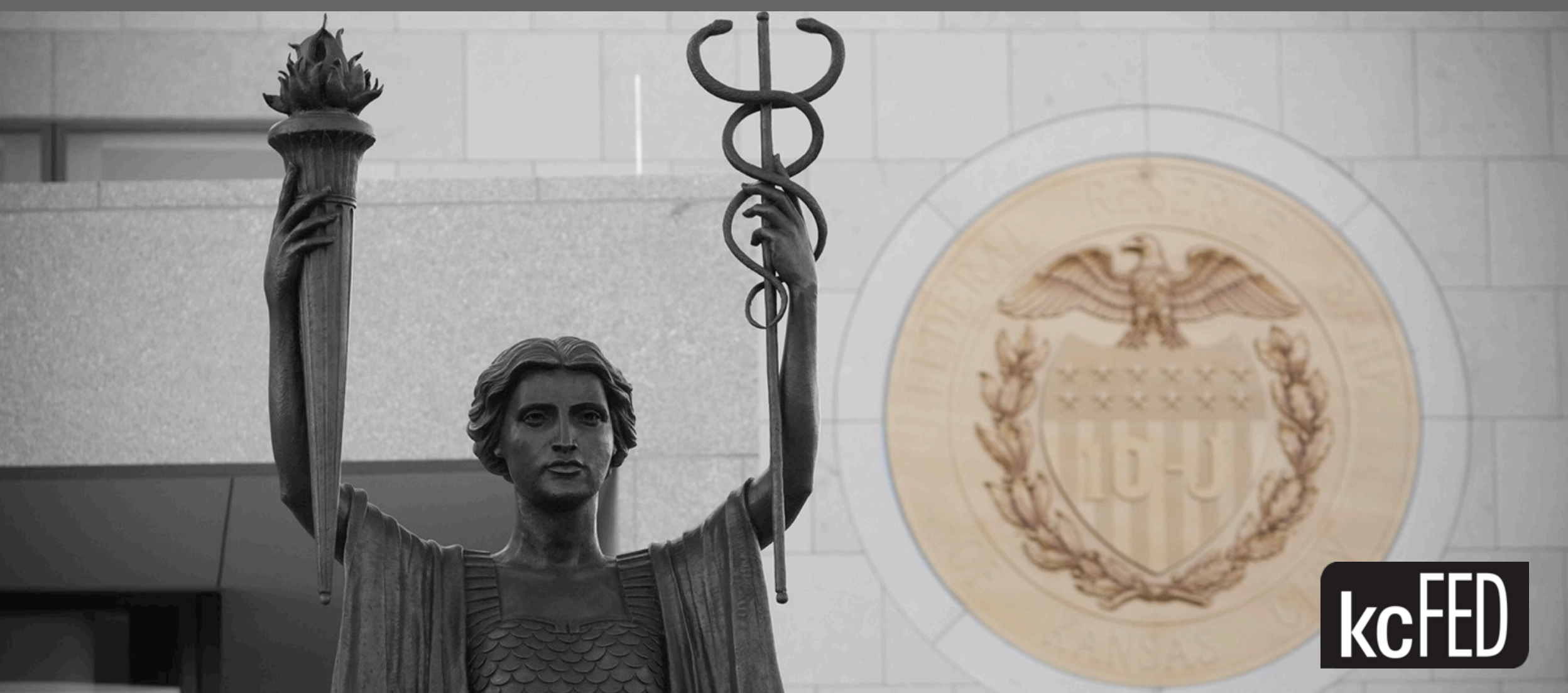


Gold (AU)DRIPSS Framework

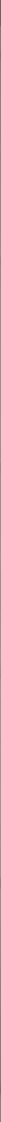




\section{Gold (AU)DRIPSS}

- Accessibility

- Usability

- Discoverability

- Reproducibility

- Interoperability

- Preservability

- Scalability

- Sustainability 


\section{Gold (AU)DRIPSS}

- Accessibility: The ability of all users, including persons with disabilities, to use the digital object

- Usability

- Discoverability

- Reproducibility

- Interoperability

- Preservability

- Scalability

- Sustainability 


\section{Gold (AU)DRIPSS}

- Accessibility

- Usability: The ability to access and tailor information to users' needs

- Discoverability

- Reproducibility

- Interoperability

- Preservability

- Scalability

- Sustainability 


\section{Gold (AU)DRIPSS}

- Accessibility

- Usability

- Discoverability: The ability to find and retrieve information

- Reproducibility

- Interoperability

- Preservability

- Scalability

- Sustainability 


\section{Gold (AU)DRIPSS}

- Accessibility

- Usability

- Discoverability

- Reproducibility: The ability to reproduce the key results of a research product or to support extensions and reanalyses

- Interoperability

- Preservability

- Scalability

- Sustainability 


\section{Gold (AU)DRIPSS}

- Accessibility

- Usability

- Discoverability

- Reproducibility

- Interoperability: A measure of the degree to which diverse systems, organizations, and/or individuals are able to work together to achieve a common goal

- Preservability

- Scalability

- Sustainability 


\section{Gold (AU)DRIPSS}

- Accessibility

- Usability

- Discoverability

- Reproducibility

- Interoperability

- Preservability: The maintenance of a continuing and uncorrupted copy of a digital object, which guarantees the public faith or truthfulness of the digital object itself

- Scalability

- Sustainability 


\section{Gold (AU)DRIPSS}

- Accessibility

- Usability

- Discoverability

- Reproducibility

- Interoperability

- Preservability

- Scalability: The ability to accommodate an increasing number of digital objects and to process growing volumes of work gracefully

- Sustainability 


\section{Gold (AU)DRIPSS}

- Accessibility

- Usability

- Discoverability

- Reproducibility

- Interoperability

- Preservability

- Scalability

- Sustainability: The ability to maintain a process or object at a desirable level of utility and continue a project's goals, principles, and efforts to achieve desired outcomes 


\section{Gold (AU)DRIPSS}

- Accessibility

- Usability

- Discoverability

- Reproducibility

- Interoperability

- Preservability

- Scalability

- Sustainability 
Examples of Balancing Priorities

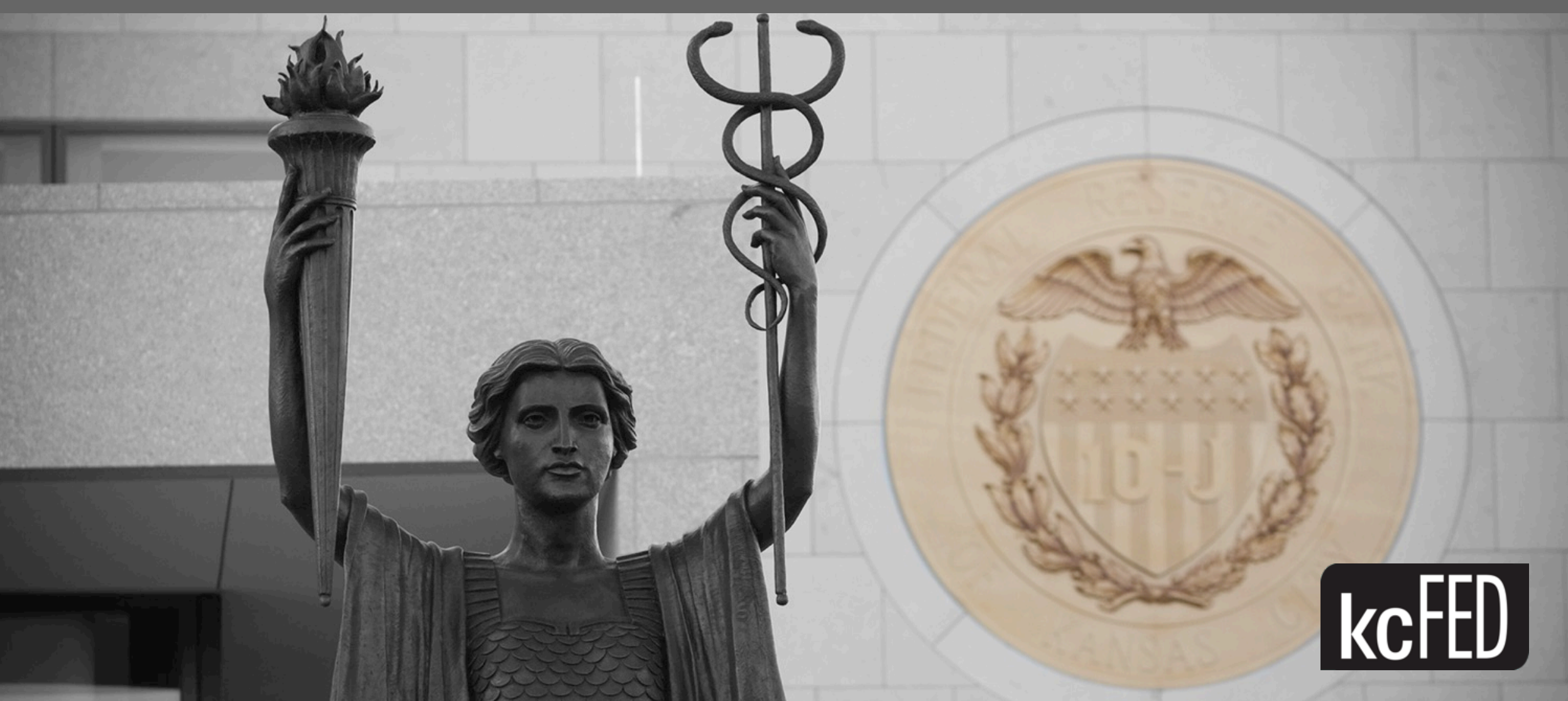




\section{Research Code}

Primary Concern

- Preservable
Secondary Concern

- Usable 


\section{More Product Less Process (MPLP)}

Primary Concern

- Scalable
Secondary Concern

- Discoverable 


\section{Example of Differing Perspectives}

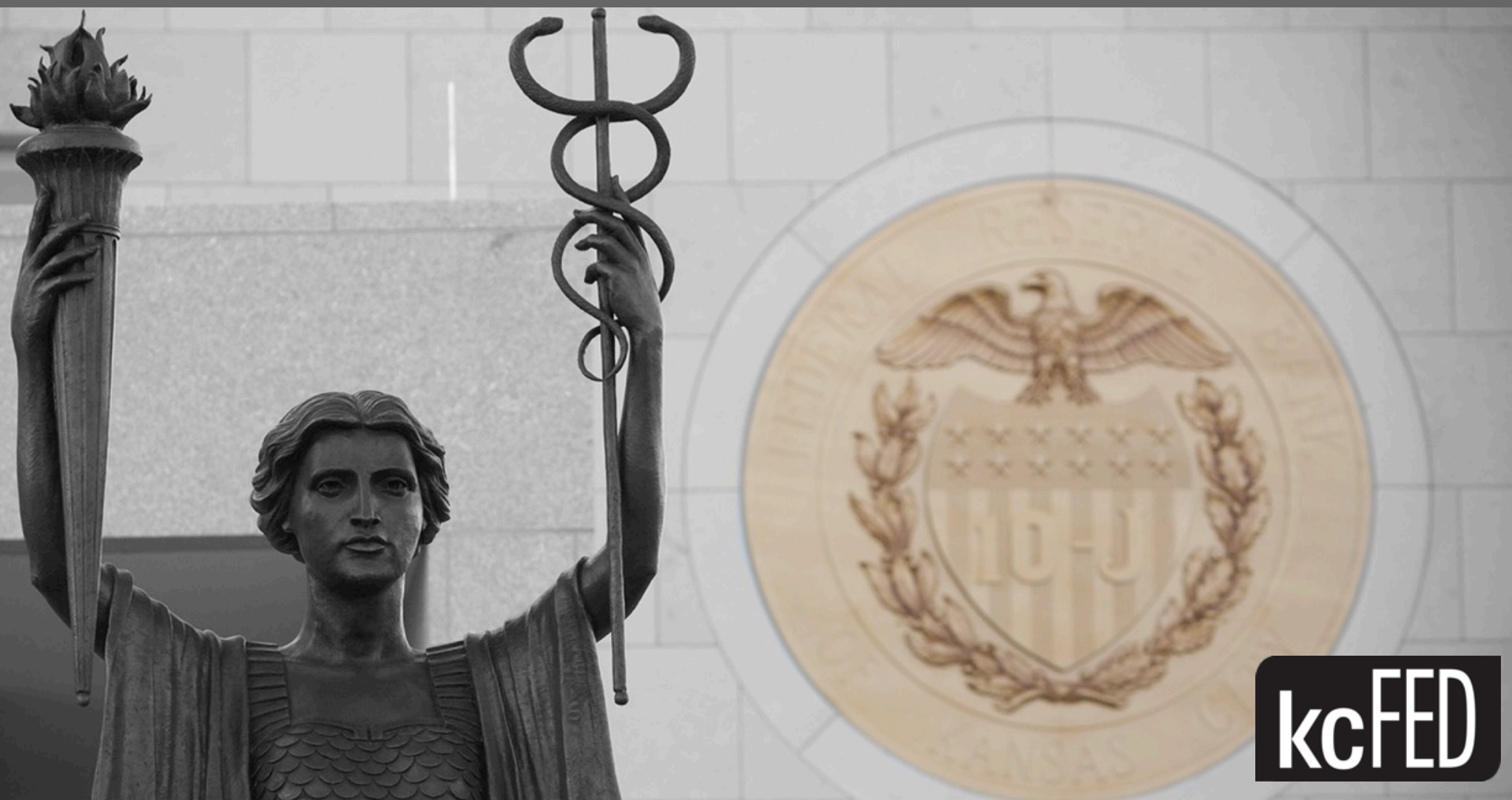




\section{Within Principle Example}

- Same Stakeholder Group

- Interoperable

- Systems?

- Schemas?
- Different Stakeholder Group

- Accessibility

- Researchers:

- Preservationists 
Rebalancing 


\section{Why rebalance?}

- Writing into project plan

- Changed your priorities

- Did not work out as intended 


\section{At Risk Items}

Primary Concern

- Preservation
Secondary Concern

- Discovery 


\section{More Product Less Process}

Primary Concerns

- Scalable

- Sustainable
Secondary Concerns

- Discoverable

- Usable 


\section{Why use?}

- Consistent definitions for stakeholders

- Model for decision making

- No right answer. Context and situation matters.

- Framework for explaining why 


\section{Questions}

